\title{
Pulmonary response to intratracheal instillation of ultrafine versus fine titanium dioxide: role of particle surface area Tina M Sager ${ }^{1,2}$, C Kommineni ${ }^{1}$ and Vincent Castranova*1
}

\author{
Address: ${ }^{1}$ Health Effects Laboratory Division, National Institute for Occupational Safety and Health, Morgantown, West Virginia, USA and \\ 2Department of Environmental Health, Harvard School of Public Health, Boston, Massachusetts, USA \\ Email: Tina M Sager - sst2@cdc.gov; C Kommineni - cdk2@cdc.gov; Vincent Castranova* - vic1@cdc.gov \\ * Corresponding author
}

Published: I December 2008

Particle and Fibre Toxicology 2008, 5:17 doi:10.1186/1743-8977-5-17

Received: 17 September 2008

Accepted: I December 2008

This article is available from: http://www.particleandfibretoxicology.com/content/5/l/l7

(c) 2008 Sager et al; licensee BioMed Central Ltd.

This is an Open Access article distributed under the terms of the Creative Commons Attribution License (http://creativecommons.org/licenses/by/2.0), which permits unrestricted use, distribution, and reproduction in any medium, provided the original work is properly cited.

\begin{abstract}
Background: The production and use of nanoparticles is growing rapidly due to the unique physical and chemical properties associated with their nano size and large surface area. Since nanoparticles have unique physicochemical properties, their bioactivity upon exposure to workers or consumers is of interest. In this study, the issue of what dose metric (mass dose versus surface area dose) is appropriate for toxicological studies has been addressed. Rats were exposed by intratracheal instillation to various doses of ultrafine or fine $\mathrm{TiO}_{2}$. At I, 7, or 42 days postexposure, inflammatory and cytotoxic potential of each particle type was compared on both a mass dosage $(\mathrm{mg} / \mathrm{rat})$ as well as an equal surface area dosage $\left(\mathrm{cm}^{2}\right.$ of particles per $\mathrm{cm}^{2}$ of alveolar epithelium) basis.
\end{abstract}

Results: The findings of the study show that on a mass basis the ultrafine particles caused significantly more inflammation and were significantly more cytotoxic than the fine sized particles. However, when doses were equalized based on surface area of particles delivered, the ultrafine particles were only slightly more inflammogenic and cytotoxic when compared to the fine sized particles. Lung burden data indicate that ultrafine $\mathrm{TiO}_{2}$ appears to migrate to the interstitium to a much greater extent than fine $\mathrm{TiO}_{2}$.

Conclusion: This study suggests that surface area of particles may be a more appropriate dose metric for pulmonary toxicity studies than mass of particles.

\section{Background}

Nanoscience and nanotechnology offer new opportunities for making superior materials for use in industrial and health applications $[1,2]$. As the field of nanotechnology vastly expands, many questions involving the effects of nanomaterials on the environment as well as human health have been raised which warrant investigation. The primary problem plaguing the field of nanotechnology is the possibility that nanoparticles can become suspended in air during production, incorporation into consumer products, and use or disposal of such products. Therefore, manufactured nanoparticles can become a component of the indoor and outdoor environments and, thus, the air we breathe [2].

A number of toxicology studies have suggested that for some classes of materials, biological response following deposition in the lungs is dependent on particle surface area $[3,4]$. Materials of interest typically possess characteristics such as low solubility, which leads to extended per- 
sistence in the lungs. These materials can be produced as ultrafine particles, which have high specific surface areas [5]. Titanium dioxide $\left(\mathrm{TiO}_{2}\right)$ is an example of a fine, low solubility particle which was considered to exhibit relatively low toxicity [6]. However, in a key study, Ferin et al. [7] demonstrated that ultrafine $\mathrm{TiO}_{2}\left(\mathrm{UFTiO}_{2}\right)$ caused more inflammation in rat lungs than exposure to the same airborne mass concentration of fine $\mathrm{TiO}_{2}\left(\mathrm{FTiO}_{2}\right)$. Up until this point in time, $\mathrm{TiO}_{2}$ often had been used as a low solubility, low toxicity, control dust in many studies. Therefore, this report was highly influential in highlighting that a material that was low in solubility and toxicity in the form of fine particles could be bioactive in the form of ultrafine particles [8].

In the last decade, it has become clear from rat studies that chronic exposure to high airborne mass concentrations of low solubility, low toxicity particles, such as carbon black and titanium dioxide, can lead to development of the features characteristic of "rat lung overload" [9]. A breakdown in normal alveolar macrophage- mediated clearance is seen in overload. This particle-induced depression of clearance was proposed to be a consequence of volumetric overload of the alveolar macrophages and the resulting loss of alveolar macrophage mobility [10]. This overload phenomenon was thought to be a problem related to particles occupying a large volume inside each macrophage and, thus, preventing them from phagocytosing additional particles and moving to the mucociliary escalator to be cleared. This was known as the volumetric overload hypothesis $[8,10]$. However, more recent studies suggest that volumetric overload is not a complete explanation for the toxicological response to ultrafine particles. Recent evidence has suggested that surface area may drive inflammation [11]. Exposure of rats to nanoparticles, with a large surface area per mass, showed features of poor clearance and inflammation at a lower lung burden, in terms of mass and volume of particles, compared with larger size particles with the same mineral and chemical properties [4].

A study conducted by Tran et al. [11] compared the inflammatory response to inhalation of two sizes of two very different types of low solubility, low toxicity particles. At an equal lung mass burden, the smaller particles were more inflammatory than the larger particles. However, when the lung burden was expressed as total particle surface area delivered, the inflammatory potential of the large and small particles was similar [11]. This supports the hypothesis that surface area of the particle may be an important component of toxic potential. In contrast, in vitro studies by Monteiller et al. [9] utilized insoluble quartz, which has a very high surface reactivity. Due to this very high surface reactivity, quartz stimulated release of inflammatory mediators at a much lower mass and surface area dose than did low solubility, low toxicity parti- cles. This is consistent with the high inflammatory potential of quartz in animal models. The outcome of this study indicates that, while surface area is important, surface activity is also a critical factor to assess pulmonary toxicity of particles. Studies supporting an important role of particle surface area in the bioactivity of low solubility, low toxicity particles, such as ultrafine carbon black and ultrafine titanium dioxide, impact assessment of workplace and environmental exposure to these nanoparticles, since regulations are currently based on mass of airborne particles [9].

In summary, several studies have concluded that on an equal mass dose basis ultrafine or nano-sized particles are uniquely more toxic than fine-sized particles of the same composition due to a high particle surface area for the nanoparticles $[4,7-9,11]$. Recently, this conclusion has been questioned $[12,13]$. It is possible that these conflicting results may be due to the use of highly agglomerated $\mathrm{UFTiO}_{2}$ samples in the later two studies $[12,13]$. Therefore, the aim of the present study was to readdress the issue of the relative toxicity of $\mathrm{UFTiO}_{2}$ vs $\mathrm{FTiO}_{2}$ on a mass dose or equivalent particle surface area delivered dose basis using alveolar lining fluid, which our laboratory has shown previously greatly improves nanoparticles dispersion [14], as the particle suspension medium. In the present study, in vivo intratracheal exposures of rats to ultrafine and fine titanium dioxide were conducted. Animals were given a dose of particles based on mass, and dose was normalized to surface area of particles administered. Particle-induced changes in several pulmonary toxicity parameters were compared on a mass dose and surface area of particles administered basis to determine which dose metric is more appropriate in nanoparticle toxicology studies.

\section{Results}

UFTiO 2 and $\mathrm{FTiO}_{2}$ suspended in acellular BALF were administered to Fischer 344 rats via intratracheal instillation to assess pulmonary toxicity. BALF was used as the suspension medium, because it greatly improves dispersion of $\mathrm{TiO}_{2}$ [14]. Figure 1 shows electron micrographs of the ultrafine (1A) and fine (1B) particles in suspension, indicating that the majority of ultrafine particles agglomerates were in the $200-300 \mathrm{~nm}$ range while the fine particles exceeded $1 \mathrm{um}$ in diameter. The dose of particles administered was expressed on a mass basis ( $\mathrm{mg} / \mathrm{rat})$ and was also normalized to surface area of particles administered per alveolar epithelial surface area $\left(\mathrm{cm}^{2} / \mathrm{cm}^{2}\right)$. The surface areas of the respective particles were determined by the BET gas absorption technique with values of 48.08 $\mathrm{m}^{2} / \mathrm{g}$ for $\mathrm{UFTiO}_{2}$ and $2.34 \mathrm{~m}^{2} / \mathrm{g}$ for $\mathrm{FTiO}_{2}$ [15]. This comparison of mass and surface area doses was conducted to assess whether surface area of particles administered is the more accurate dose metric that should be considered when assessing nanoparticle pulmonary toxicity parame- 

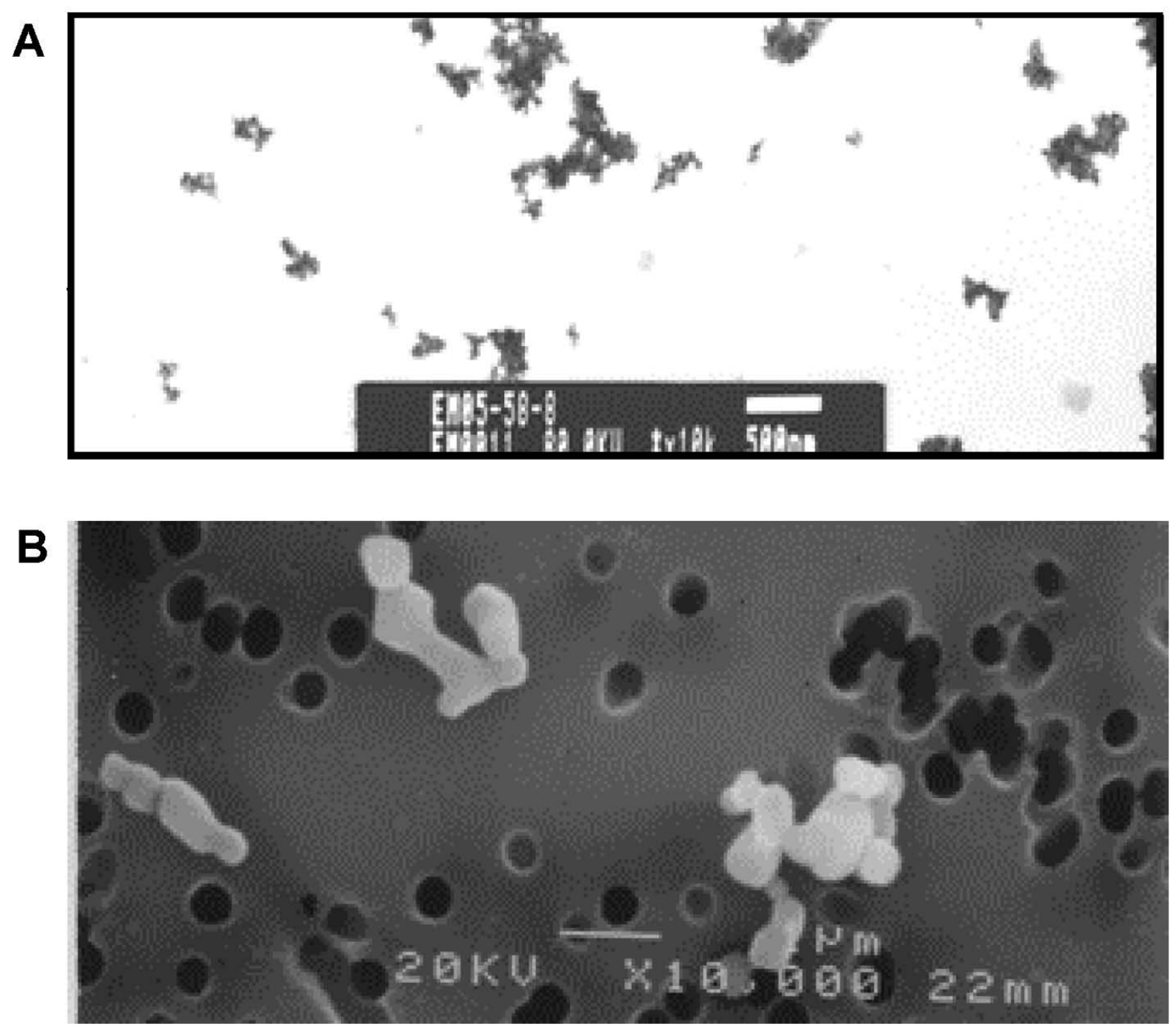

Figure I

Electron micrographs of titanium dioxide particles suspended in BALF. A transmission electron micrograph of $\mathrm{UFTiO}_{2}$ suspended in BALF (A). Magnification of $60,000 \times$ (note $500 \mathrm{~nm}$ scale bar). A scanning electron micrograph of $\mathrm{FTiO}_{2}$ suspended in BALF (B). Magnification of 20,000 $\times$ (note I um scale bar).

ters. Pulmonary response parameters measured included, indicators of pulmonary inflammation (PMN number or inflammatory mediators, such as TNF- $\alpha$, MIP-2, and IL$1 \beta$ ), markers of lung injury (LDH activity or albumin levels) and markers of macrophage activity (zymosan-stimulated chemiluminescence and NO-dependent chemiluminescence).

\section{Comparison of pulmonary toxicity of $\mathrm{UFTiO}_{2}$ and $\mathrm{FTiO}_{2}$}

On a mass dose basis, $\mathrm{UFTiO}_{2}$ and $\mathrm{FTiO}_{2}$ both produced a dose-dependent increase in PMN harvested by BAL (Figure 2). All doses of $\mathrm{UFTiO}_{2}$ caused a significant increase in
PMN over control at all post-exposure time periods. For $\mathrm{FTiO}_{2}$, the medium and high doses caused a significant increase in PMN number over control at all post-exposure days. However, it took a significantly larger mass dose of $\mathrm{FTiO}_{2}$ to obtain the same inflammogenic response as the $\mathrm{UFTiO}_{2}$ particles (Figure 2). When comparing this inflammogenic response for $\mathrm{UFTiO}_{2}$ exposure to $\mathrm{FTiO}_{2}$ exposure, on a mass dose basis (for example the PMN response to $10.7 \mathrm{mg} / \mathrm{rat} \mathrm{FTiO}_{2}$ vs $0.26 \mathrm{mg} / \mathrm{rat} \mathrm{UFTiO}_{2}$ from figure 2), $\mathrm{UFTiO}_{2}$ was shown to be 41 times more potent than the $\mathrm{FTiO}_{2}$ at all exposure times (Table 1). However, when dose of particles was normalized to surface area of parti- 
cles administered the difference in inflammogenic responses, assessed by PMN number, of the two particle types was much less (Figure 3). Indeed, a linear regression curve analysis with a $95 \%$ confidence interval showed that there was no significant difference between the doseresponse curves for $\mathrm{UFTiO}_{2}$ and $\mathrm{FTiO}_{2}$ when dose was expressed as total particle surface area delivered (Figure 3 ). In fact, when dose was normalized to surface area of particles administered, the inflammogenic response elicited by $\mathrm{UFTiO}_{2}$ was only 2 -fold greater than the $\mathrm{FTiO}_{2}$ response at 1 day post-exposure, 1.3 -fold greater at 7 days post-exposure and 1.6-fold greater at 42 days post-exposure (Table 1).

Lactate dehydrogenase (LDH) activity was measured to assess cellular cytotoxicity after $\mathrm{TiO} 2$ exposures. As with PMN, both UFTiO2 and FTiO2 caused a dose dependent increase in LDH activity in BALF. For LDH activity, on a mass dose basis, a significantly greater mass dose of FTiO2 was required at all post-exposure time points to obtain the same responses as seen with UFTiO2 exposure (Figure 4). For LDH activity, on a mass dose basis, UFTiO2 exposure was shown to be approximately 30-41 times more potent at all post-exposure time points than $\mathrm{FTiO} 2$ exposure (Table 1). However, when dose of particles was normalized to surface area of particles administered the difference in LDH activity of the two particle types was much less (Figure 5). Indeed, a linear regression curve analysis with a 95\% confidence interval showed that there was no significant difference between the dose-response curves for UFTiO2 and FTiO2 when dose was expressed as total particle surface area delivered (Figure 5). In fact, when dose was normalized to surface area of particles administered, the UFTiO2 exposure produced LDH activity levels that were at most only 1.8 -fold greater than the FTiO2 exposure at all post-exposure time points analyzed (Table $1)$.

Table I: Potency difference between $\mathrm{UFTiO}_{2}$ and $\mathrm{FTiO}_{2}$ when analyzed on a mass vs. surface area basis.

\begin{tabular}{|c|c|c|c|c|}
\hline Parameter & Dose Metric & I Day & 7 Days & 42 Days \\
\hline \multicolumn{5}{|l|}{ PMN (106 $)$} \\
\hline & Mass & 41 & 41 & 41 \\
\hline & Surface Area & 2 & 1.3 & 1.6 \\
\hline \multicolumn{5}{|l|}{ LDH (U/I) } \\
\hline & Mass & 41 & 30 & 41 \\
\hline & Surface Area & 1.2 & 1.2 & 1.8 \\
\hline \multicolumn{5}{|l|}{ Albumin } \\
\hline & Mass & 82 & 41 & $4 I$ \\
\hline & Surface Area & 3 & 1.4 & 2 \\
\hline \multicolumn{5}{|c|}{ TNF- $\alpha(\mathrm{pg} / \mathrm{ml})$} \\
\hline & Mass & 41 & 41 & 41 \\
\hline & Surface Area & 1.2 & 1.4 & 1.5 \\
\hline \multicolumn{5}{|c|}{ MIP-2 (pg/ml) } \\
\hline & Mass & 41 & 41 & 82 \\
\hline & Surface Area & 1.2 & 1.1 & 1.1 \\
\hline \multicolumn{5}{|c|}{$\underline{\mathrm{IL}-\mathrm{I} \beta(\mathrm{pg} / \mathrm{ml})}$} \\
\hline & Mass & 82 & 82 & 82 \\
\hline & Surface Area & 1.6 & 1.8 & 1.4 \\
\hline \multicolumn{5}{|c|}{ Zym. Stim. Chemi. } \\
\hline & Mass & 82 & 82 & 82 \\
\hline & Surface Area & 1.75 & 1.8 & 3 \\
\hline \multicolumn{5}{|c|}{ NO Dep. Chemi. } \\
\hline & Mass & 41 & 82 & 82 \\
\hline & Surface Area & 1.5 & 7 & 8 \\
\hline
\end{tabular}

Comparison of $\mathrm{UFTiO}_{2}$ and $\mathrm{FTiO}_{2}$ potency differences. The data were analyzed to show the potency difference between $\mathrm{UFTiO}_{2}$ and $\mathrm{FTiO}_{2}$ on a mass basis as well as the fold increase in pulmonary toxicity response on a surface area basis. All post-exposure time points were analyzed and are reported in the table. On a mass basis, the $\mathrm{UFTiO}_{2}$ has much greater potency than $\mathrm{FTiO}_{2}$, but when dose is normalized to total particle surface area administered the fold increase in response between the $\mathrm{UFTiO}_{2}$ and $\mathrm{FTiO}_{2}$ is greatly reduced. 
$\underline{\text { A. }}$

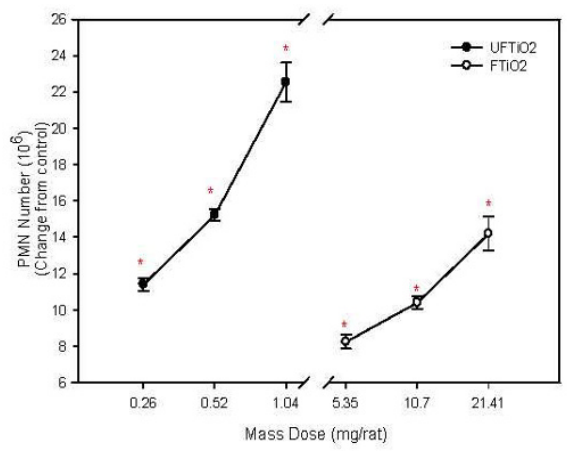

B.

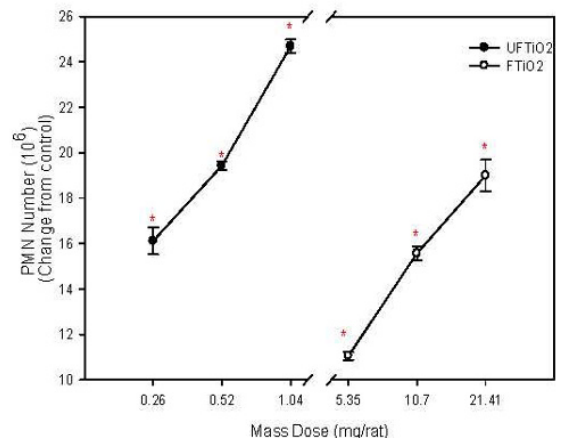

C.

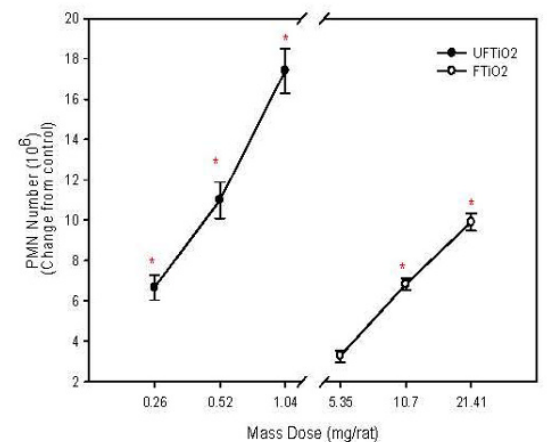

Figure 2

A comparison of inflammation elicited in animals receiving various mass doses of $\mathrm{UFTiO}_{2}$ and $\mathrm{FTiO}_{2}$ suspended in BALF. A comparison of inflammation elicited in animals receiving various mass doses of $\mathrm{UFTiO}_{2}$ and $\mathrm{FTiO}_{2}$ suspended in BALF at I day (Panel A), 7 days (Panel B), and 42 days (Panel C) post-exposure. Rats were exposed to various mass doses of $\mathrm{UFTiO}_{2}$ and $\mathrm{FTiO}_{2}$ by intratracheal instillation. Animals were euthanized at I day, 7 days, and 42 days post-exposure and bronchoalveolar lavage was performed. Inflammation was assessed by BAL PMN counts. Values are increased PMN number above the BALF control and are given as means \pm SE of 8 experiments. Control PMN values were $1.37 \pm 0.098 \times 10^{6}$, $0.78 \pm 0.074 \times 10^{6}$, and $0.88 \pm 0.095 \times 10^{6}$ cells/rat for I, 7 and 42 days, respectively. Linear regression analysis with a $95 \%$ confidence interval reveals that on a mass dose basis $\mathrm{UFTiO}_{2}$ causes significantly more inflammation than $\mathrm{FTiO}_{2}$ at all post-exposure time points. $*$ indicates a significant increase from control $(\mathrm{p}<0.05$; ANOVA).

$\underline{\mathbf{A}}$

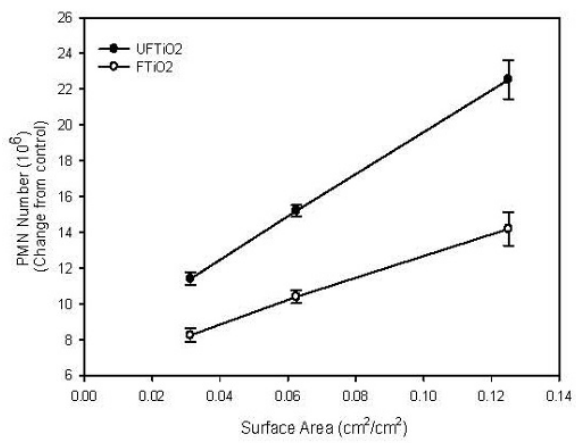

B.

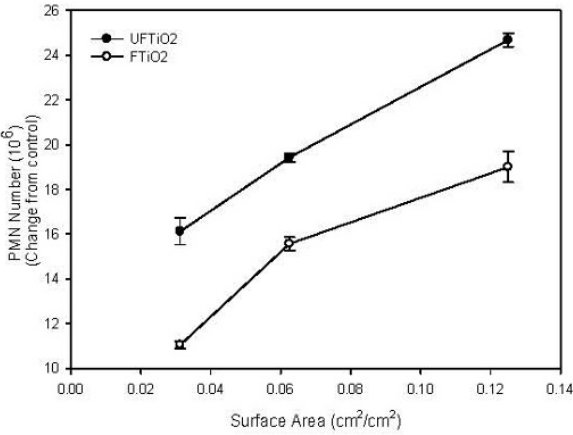

C.

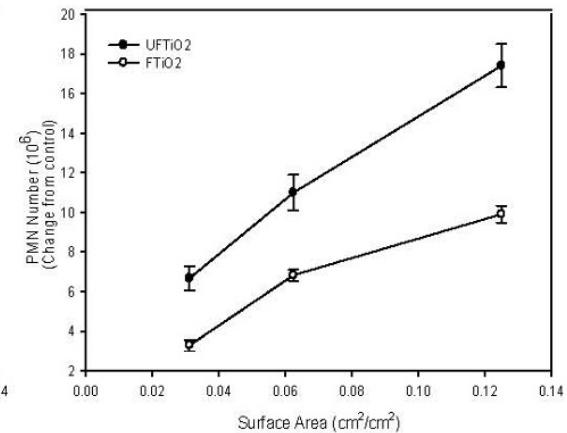

Figure 3

A comparison of inflammation elicited in animals receiving doses of $\mathrm{UFTiO}_{2}$ and $\mathrm{FTiO}_{2}$ normalized to surface area of particles administered per surface area of alveolar epithelium. A comparison of inflammation elicited in animals receiving doses $\left(0.0313,0.0625\right.$ and $\left.0.125 \mathrm{~cm}^{2} / \mathrm{cm}^{2}\right)$ of $\mathrm{UFTiO}_{2}$ and $\mathrm{FTiO}_{2}$ normalized to surface area of particles administered per surface area of alveolar epithelium at I day (Panel A), 7 days (Panel B), and 42 days (Panel C) post-exposure. Particles were suspended in BALF. Rats were exposed to various doses of $\mathrm{UFTiO}_{2}$ and $\mathrm{FTiO}_{2}$ by intratracheal instillation. Animals were euthanized at I day, 7 days, and 42 days post-exposure and bronchoalveolar lavage was performed. Inflammation was assessed by BAL PMN counts. Values are increased PMN number above the BALF control and are given as means \pm SE of 8 experiments. Control PMN values were $1.37 \pm 0.098 \times 10^{6}, 0.78 \pm 0.074 \times 10^{6}$, and $0.88 \pm 0.095 \times 10^{6}$ cells/rat for I, 7 and 42 days, respectively. Linear regression analysis with a $95 \%$ confidence interval reveals that, when dose is normalized to surface area of particles administered, dose responses curves assessing inflammation caused by $\mathrm{UFTiO}_{2}$ and $\mathrm{FTiO}_{2}$ exposure are not significantly different. On a dose normalized to surface area, $\mathrm{UFTiO}_{2}$ elicits at most a 2 fold increase in inflammation when compared to $\mathrm{FTiO}_{2}$ at all post-exposure times. 
$\underline{\text { A. }}$

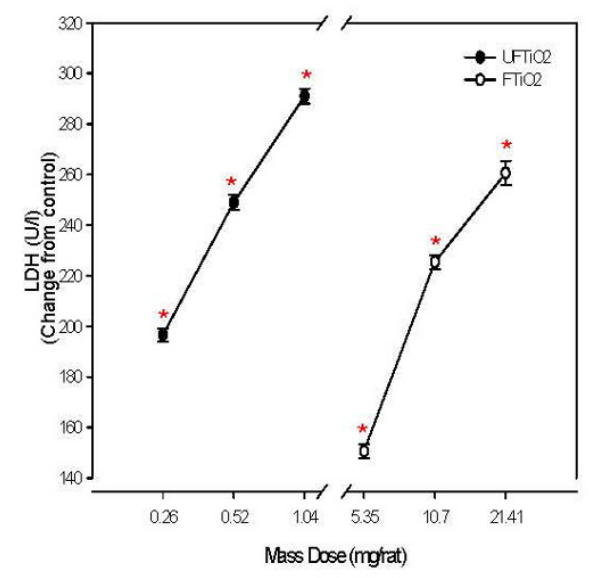

B.

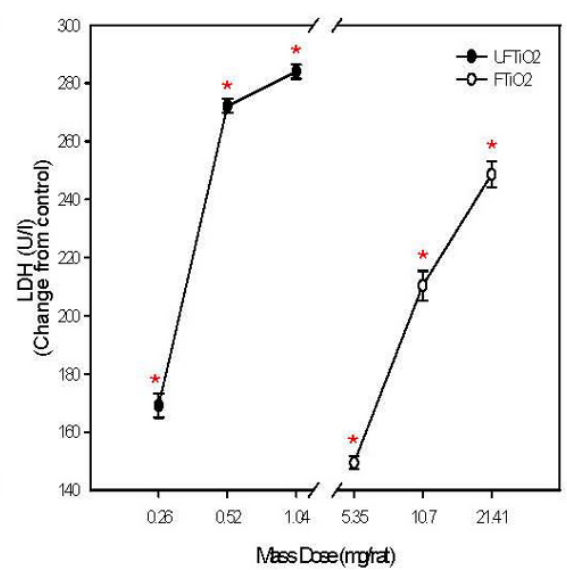

C.

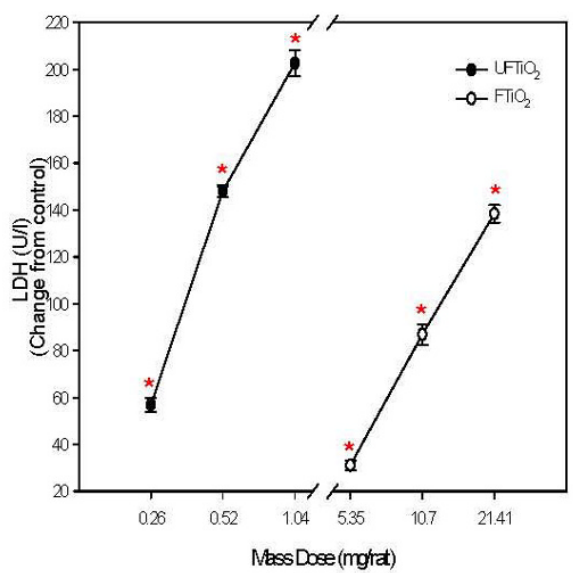

Figure 4

A comparison of cellular cytotoxicity elicited in animals receiving various mass doses of $\mathrm{UFTiO}_{2}$ and $\mathrm{FTiO}_{2}$ suspended in BALF. A comparison of cellular cytotoxicity elicited in animals receiving various mass doses of $\mathrm{UFTiO}_{2}$ and $\mathrm{FTiO}_{2}$ suspended in BALF at I day (Panel A), 7 days (Panel B), and 42 days (Panel C) post-exposure. Rats were exposed to various mass doses of $\mathrm{UFTiO}_{2}$ and $\mathrm{FTiO}_{2}$ by intratracheal instillation. Animals were euthanized at I day, 7 days, and 42 days post-exposure and bronchoalveolar lavage was performed. Cytotoxicity was assessed by measuring LDH activity. Values are increase in LDH activity above the BALF control and are given as means \pm SE of 8 experiments. Control values of LDH activity were $46.375 \pm 2.24,39.5 \pm 1.35$, and $37.25 \pm 2.63 \mathrm{U} / \mathrm{l}$ for I, 7 and 42 days, respectively. Linear regression analysis with a $95 \%$ confidence interval reveals that on a mass dose basis $\mathrm{UFTiO}_{2}$ causes significantly more $\mathrm{LDH}$ activity than $\mathrm{FTiO}_{2}$ at all post-exposure time points. $*$ indicates a significant increase from control ( $p<0.05$; ANOVA).

$\underline{\text { A. }}$

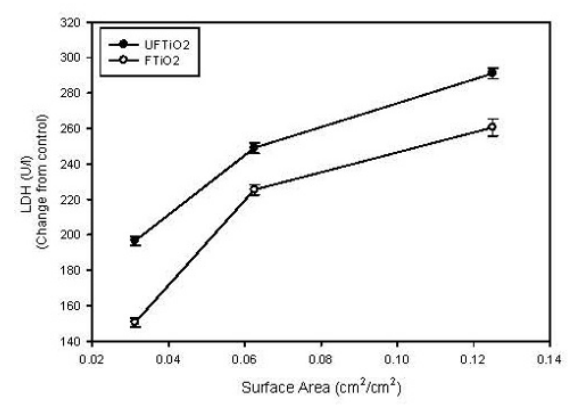

B.

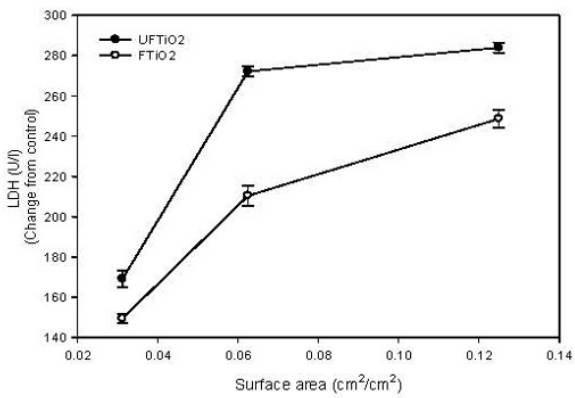

C.

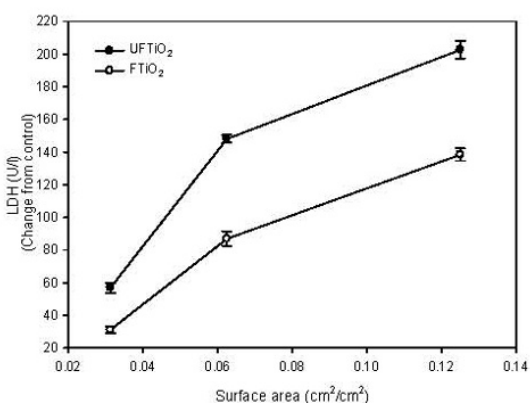

\section{Figure 5}

A comparison of cytotoxicity elicited in animals receiving doses of $\mathrm{UFTiO}_{2}$ and $\mathrm{FTiO}_{2}$ normalized to surface area of particle administered per surface area of alveolar epithelium. A comparison of cytotoxicity elicited in animals receiving doses $\left(0.0313,0.0625\right.$ and $\left.0.125 \mathrm{~cm}^{2} / \mathrm{cm}^{2}\right)$ of $\mathrm{UFTiO}_{2}$ and $\mathrm{FTiO}_{2}$ normalized to surface area of particles administered per surface area of alveolar epithelium at I day (Panel A), 7 days (Panel B), and 42 days (Panel C) post-exposure. Animals were exposed to various doses of $\mathrm{UFTiO}_{2}$ and $\mathrm{FTiO}_{2}$ by intratracheal instillation. Animals were euthanized at I day, 7 days, and 42 days post-exposure and bronchoalveolar lavage was performed. Cytotoxicity was assessed by measuring LDH activity. Values are increase in LDH activity above the BALF control and are given as means \pm SE of 8 experiments. Control values of LDH activity were $46.375 \pm 2.24,39.5 \pm \mathrm{I} .35$, and $37.25 \pm 2.63 \mathrm{U} / \mathrm{l}$ for I, 7 and 42 days, respectively. Linear regression analysis with $95 \%$ confidence internal reveals that, when dose is normalized to surface area of particles administered, responses to $\mathrm{UFTiO}_{2}$ and $\mathrm{FTiO}_{2}$ are not significantly different. 
Albumin levels in BALF were analyzed to assess air/blood barrier injury after $\mathrm{TiO}_{2}$ exposures. Both $\mathrm{UFTiO}_{2}$ and $\mathrm{FTiO}_{2}$ caused a dose dependent increase in albumin levels. For albumin levels, on a mass dose basis, a significantly greater mass dose of $\mathrm{FTiO}_{2}$ was required at all post-exposure time points, to obtain the same responses as seen with $\mathrm{UFTiO}_{2}$ exposure (Figure 6). In fact, for albumin levels, $\mathrm{UFTiO}_{2}$ exposure was shown to be approximately 82 times more potent than $\mathrm{FTiO}_{2}$ at 1 day post-exposure and 41 times more potent at 7 and 42 days post-exposure when mass was the dose metric (Table 1). However, when dose of particles was normalized to surface area of particles administered the difference in albumin levels of the two particle types was much less (Figure 7). Indeed, a linear regression curve analysis with a 95\% confidence interval showed that there was no significant difference between the dose-response curves for $\mathrm{UFTiO}_{2}$ and $\mathrm{FTiO}_{2}$ when dose was expressed as total particle surface area delivered (Figure 7). When dose was normalized to surface area of particles administered, the $\mathrm{UFTiO}_{2}$ exposure produced albumin levels that were at most only 3 fold greater than the $\mathrm{FTiO}_{2}$ exposure at all post-exposure time points analyzed (Table 1).

IL-1 $\beta$, TNF- $\alpha$, and MIP-2 levels were also measured in response to various mass doses of $\mathrm{UFTiO}_{2}$ and $\mathrm{FTiO}_{2}$ at all post-exposure time points (data similar to Figures 2, 4 and 6 not shown). The highest dose of $\mathrm{UFTiO}_{2}$ caused a signif- icant increase in TNF- $\alpha$, IL-1 $\beta$ and MIP- 2 mediator levels over control values at all post-exposure time points (Table 2). The high dose of $\mathrm{FTiO}_{2}$ caused a significant increase in MIP-2 and TNF- $\alpha$ levels at all post-exposure time points and caused a significant increase in IL-1 $\beta$ cytokine levels at 7 days and 42 days post-exposure (Table 2). For all three mediators, a significantly greater mass dose of $\mathrm{FTiO}_{2}$ was required to obtain the comparable levels in the BAL fluid as elicited by $\mathrm{UFTiO}_{2}$ exposure (Table 1). For TNF- $\alpha$ cytokine levels, on a mass dose basis, $\mathrm{UFTiO}_{2}$ was shown to be 41 times more potent than $\mathrm{FTiO}_{2}$ at all post-exposure time points (Table 1 ). However, when dose was normalized to surface area of particles administered, TNF- $\alpha$ cytokine levels produced by $\mathrm{UFTiO}_{2}$ exposure were at most only 1.5 -fold greater than $\mathrm{FTiO}_{2}$ levels. For MIP-2 levels, on a mass dose basis, $\mathrm{UFTiO}_{2}$ was shown to be 41 times more potent than $\mathrm{FTiO}_{2}$ at 1 and 7 days post-exposure and 82 times more potent at 42 days post-exposure (Table 1). However, when dose was normalized to surface area of particles administered, MIP-2 levels produced by $\mathrm{UFTiO}_{2}$ exposure were at most only 1.2 -fold greater than $\mathrm{FTiO}_{2}$ levels (Table 1 ). In regards to IL- $1 \beta$ cytokine levels, on a mass dose basis, $\mathrm{UFTiO}_{2}$ was shown to be 82 times more potent than $\mathrm{FTiO}_{2}$ at all post-exposure time points (Table 1). However, when dose was normalized to surface area of particles administered, IL-1 $\beta$ cytokine levels produced by $\mathrm{UFTiO}_{2}$ exposure were at most only 1.8 -fold greater than $\mathrm{FTiO}_{2}$ levels (Table 1). Dose response curves
$\underline{\mathbf{A}}$

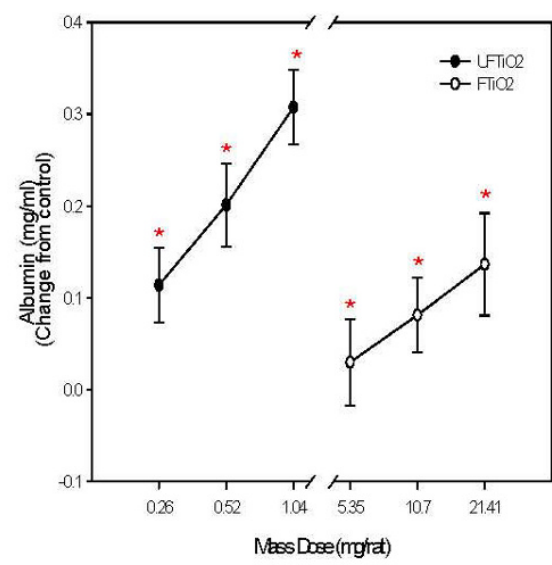

B.

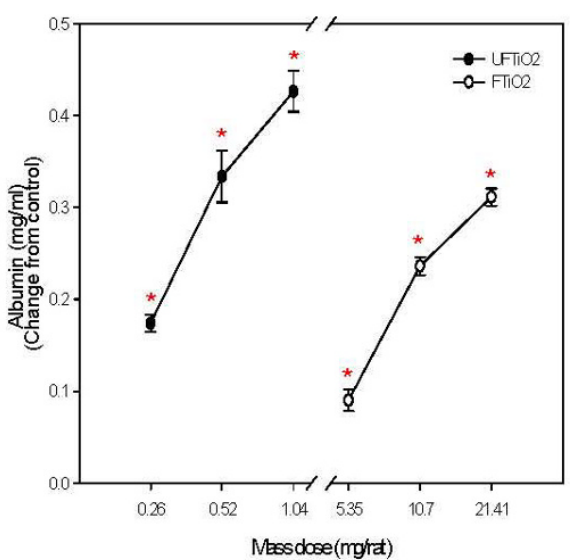

$\underline{\text { C. }}$

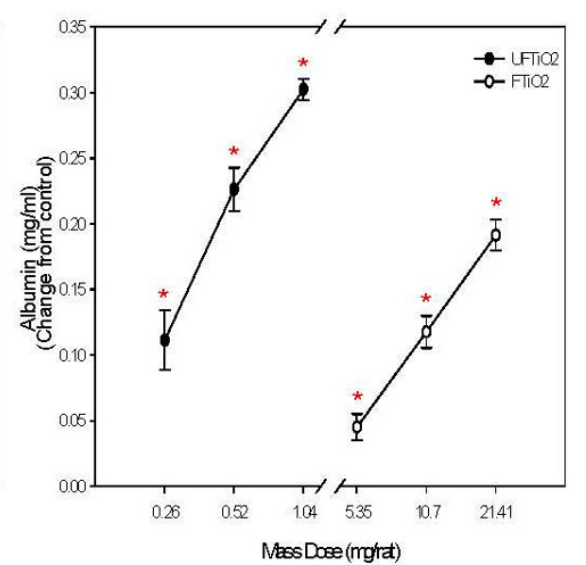

Figure 6

A comparison of cellular damage elicited in animals receiving various mass doses of $\mathrm{UFTiO}_{2}$ and $\mathrm{FTiO}_{2}$ suspended in BALF. A comparison of cellular damage elicited in animals receiving various mass doses of $\mathrm{UFTiO}_{2}$ and $\mathrm{FTiO}_{2}$ suspended in BALF at I day (Panel A), 7 days (Panel B), and 42 days (panel C) post-exposure. Rats were exposed to various mass doses of $\mathrm{UFTiO}_{2}$ and $\mathrm{FTiO}_{2}$ by intratracheal instillation. Animals were euthanized at I day, 7 days, and 42 days post-exposure and bronchoalveolar lavage was performed. Cellular injury was assessed by measuring albumin levels. Control values of albumin levels were $0.073 \pm 0.033,0.084 \pm 0.003,0.098 \pm 0.007 \mathrm{mg} / \mathrm{ml}$ for I, 7, and 42 days, respectively. Values are increase in albumin levels above the BALF control and are given as means $\pm S E$ of 8 experiments. * indicates a significant increase from control ( $P$ $<0.05$; ANOVA). 
$\underline{\mathbf{A}}$

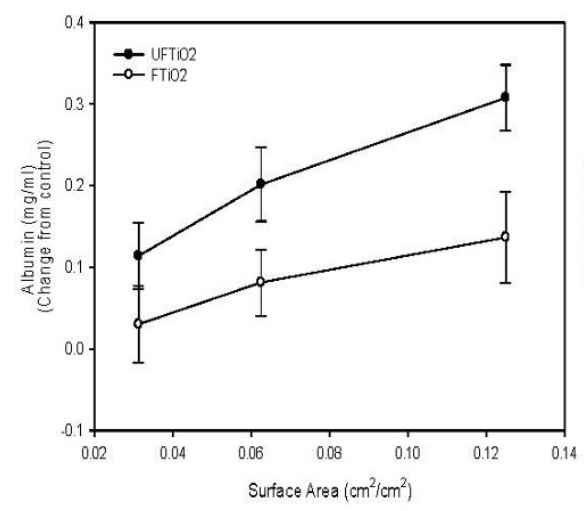

B.

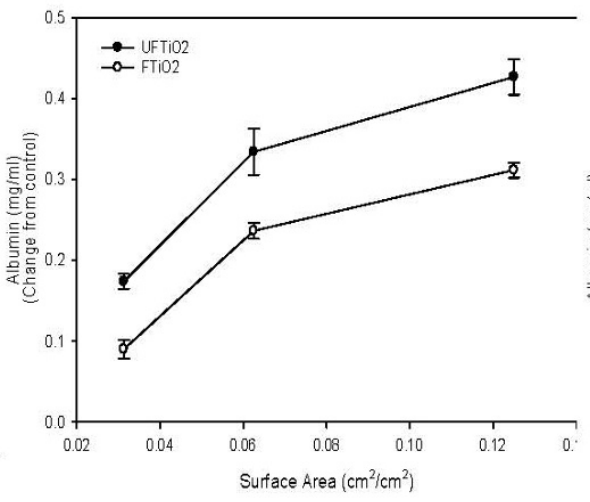

C.

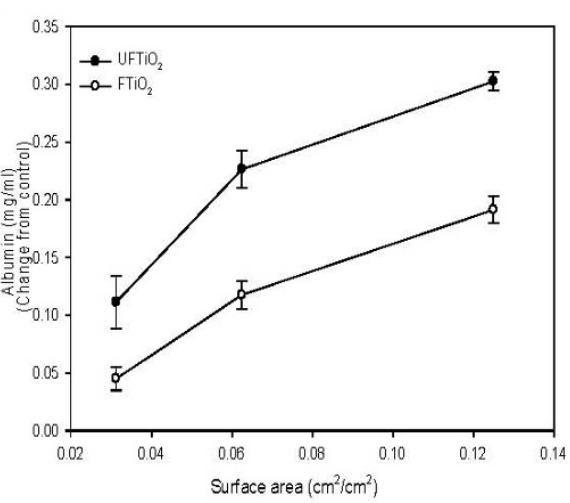

Figure 7

A comparison of cellular damage elicited in animals receiving doses of $\mathrm{UFTiO}_{2}$ and $\mathrm{FTiO}_{2}$ normalized to surface area of particle administered per surface area of alveolar epithelium. A comparison of cellular damage elicited in animals receiving doses $\left(0.0313,0.0625\right.$ and $\left.0.125 \mathrm{~cm}^{2} / \mathrm{cm}^{2}\right)$ of UFTiO2 and $\mathrm{FTiO}_{2}$ normalized to surface area of particles administered per surface area of alveolar epithelium at I day (Panel A), 7 days (Panel B), and 42 days (Panel C) post-exposure. Rats were exposed to various mass doses of $\mathrm{UFTiO}_{2}$ and $\mathrm{FTiO}_{2}$ by intratracheal instillation. Animals were euthanized at $\mathrm{I}$ day, 7 days, and 42 days post-exposure and bronchoalveolar lavage was performed. Cellular injury was assessed by measuring albumin levels. Control values of albumin levels were $0.073 \pm 0.033,0.084 \pm 0.003,0.098 \pm 0.007 \mathrm{mg} / \mathrm{ml}$ for $\mathrm{I}, 7$, and 42 days, respectively. Values are increase in albumin levels above the BALF control and are given as means \pm SE of 8 experiments. Linear regression analysis with a $95 \%$ confidence interval reveals that, when dose is normalized to surface area of particles administered, responses to $\mathrm{UFTiO}_{2}$ and $\mathrm{FTiO}_{2}$ are not significantly differently.

assessing TNF- $\alpha$, IL-1 $\beta$, and MIP-2 levels based on surface area of particles administered between $\mathrm{UFTiO}_{2}$ and $\mathrm{FTiO}_{2}$ exposure were assessed using a linear regression curve analysis with a $95 \%$ confidence interval (data similar to Figures 3, 5 and 7 not shown). This analysis showed there was no significant difference in the potency of $\mathrm{UFTiO}_{2}$ vs $\mathrm{FTiO}_{2}$ for any of the mediators between the two curves at any post-exposure time point.

Alveolar macrophage zymosan-stimulated and NOdependent chemiluminescence were measured to assess reactive oxygen species production after $\mathrm{UFTiO}_{2}$ and $\mathrm{FTiO}_{2}$ exposures. On a mass dose basis, $\mathrm{UFTiO}_{2}$ and $\mathrm{FTiO}_{2}$ both caused dose-dependent increases in zymosan-stimulated and NO dependent chemiluminescence (data analogous to Figures 2, 4 and 6 not shown). The responses to the high dose are shown in Table 2. A significantly greater mass dose of $\mathrm{FTiO}_{2}$ was required to produce comparable chemiluminescence levels as elicited by $\mathrm{UFTiO}_{2}$ exposure. The highest doses of $\mathrm{UFTiO}_{2}$ caused a significant increase in zymosan and NO-dependent chemiluminescence levels over control at all post-exposure time points (Table 2). The high dose of $\mathrm{FTiO}_{2}$ caused a significant increase over control in zymosan-stimulated chemiluminescence at 1 and 7 days post-exposure and in NO-dependent chemiluminecence only at 1 day post-exposure (Table 2 ).
On a mass dose basis, in regards to zymosan-stimulated chemiluminescence levels, $\mathrm{UFTiO}_{2}$ was shown to be 82 times more potent than $\mathrm{FTiO}_{2}$ at all post-exposure time points (Table 1). However, when dose was normalized to surface area of particles administered, $\mathrm{UFTiO}_{2}$ zymosanstimulated chemiluminecence levels were at most only 3 fold higher than $\mathrm{FTiO}_{2}$ zymosan-stimulated chemiluminecence levels (Table 1 ). When the surface area zymosanstimulated chemiluminescence dose response curves were analyzed using a linear regression curve with a 95\% confidence interval (data not shown), there was no significant difference between the $\mathrm{UFTiO}_{2}$ and $\mathrm{FTiO}_{2}$ dose response curves at 1 day or 7 days post-exposure.

On a mass dose basis, in regards to NO-dependent chemiluminescence levels, $\mathrm{UFTiO}_{2}$ was 41 times more potent than $\mathrm{FTiO}_{2}$ at 1 day post-exposure and was 82 times more potent at 7 and 42 days post-exposure. However, when dose was normalized to surface area of particles administered, $\mathrm{UFTiO}_{2} \mathrm{NO}$-dependent chemiluminescence levels were only 1.5 -fold greater than $\mathrm{FTiO}_{2}$ at 1 day post-exposure but were 8 -fold greater at 42 days post-exposure (Table 1). When the surface area NO-dependent chemiluminescence dose response curves were analyzed using a linear regression curve with a $95 \%$ confidence interval (data not shown), there was no significant difference 
Table 2: Effect of Exposure to $\mathrm{UFTiO}_{2}$ vs. $\mathrm{FTiO}_{2}$ (High Dose) on Pulmonary Responses.

\begin{tabular}{|c|c|c|c|c|}
\hline Parameter & Group & I day & 7 days & $\underline{42 \text { days }}$ \\
\hline \multirow[t]{3}{*}{ PMN (106) } & Control & $1.4 \pm 0.1$ & $0.78 \pm 0.07$ & $0.88 \pm 0.10$ \\
\hline & UFTiO2 high & $23.8 \pm I^{*}$ & $25.5 \pm 3 *$ & $18.3 \pm I^{*}$ \\
\hline & FTiO $_{2}$ high & $15.6 \pm 0.9 *$ & $19.8 \pm 0.7^{*}$ & $10.8 \pm 0.4^{*}$ \\
\hline \multirow[t]{3}{*}{ LDH (U/I) } & Control & $46.3 \pm 2$ & $39.5 \pm 1.35$ & $37.3 \pm 2$ \\
\hline & UFTiO2 high & $337.4 \pm 3 *$ & $323.5 \pm 3^{*}$ & $239.9 \pm 5^{*}$ \\
\hline & FTiO $_{2}$ high & $307.6 \pm 5^{*}$ & $288.1 \pm 5^{*}$ & $175.6 \pm 4^{*}$ \\
\hline \multirow[t]{3}{*}{ Albumin } & Control & $0.07 \pm 0.03$ & $0.08 \pm 0.003$ & $0.09 \pm 0.007$ \\
\hline & $\overline{\text { UFTiO2 high }}$ & $0.38 \pm 0.04^{*}$ & $0.51 \pm 0.02 *$ & $0.40 \pm 0.01 *$ \\
\hline & $\overline{\text { FTiO }}_{2}$ high & $0.21 \pm 0.055^{*}$ & $0.40 \pm 0.01 *$ & $0.30 \pm 0.01 *$ \\
\hline \multirow[t]{3}{*}{ TNF- $\alpha$ (pg/ml) } & Control & $25.7 \pm 0.6$ & $23.1 \pm 0.7$ & $25.86 \pm 0.8$ \\
\hline & $\overline{\text { UFTiO2 high }}$ & $67.4 \pm 2.2^{*}$ & $73.5 \pm 1.5^{*}$ & $88.5 \pm 4.4^{*}$ \\
\hline & FTiO $_{2}$ high & $60.7 \pm 1.7^{*}$ & $58.5 \pm 2.8^{*}$ & $55.4 \pm 1.3^{*}$ \\
\hline \multirow[t]{3}{*}{ MIP-2 (pg/ml) } & Control & $517.9 \pm 12$ & $601.8 \pm 26$ & $643.6 \pm 7$ \\
\hline & $\overline{\text { UFTiO2 high }}$ & $802.9 \pm 28^{*}$ & $886.9 \pm 21^{*}$ & $893.5 \pm 26^{*}$ \\
\hline & FTiO $_{2}$ high & $695.6 \pm 15^{*}$ & $847.1 \pm 43^{*}$ & $852.1 \pm 22 *$ \\
\hline \multirow[t]{3}{*}{$\underline{\mathrm{LL}-\mathrm{I} \beta}(\mathrm{pg} / \mathrm{ml})$} & Control & $75.9 \pm 6$ & $82.2 \pm 6$ & $91.9 \pm 8$ \\
\hline & UFTiO2 high & $167.5 \pm 13^{*}$ & $230.4 \pm 14^{*}$ & $273.6 \pm 14^{*}$ \\
\hline & FTiO $_{2}$ high & $119.9 \pm 13$ & $|3| .5 \pm\left. 1\right|^{*}$ & $180.3 \pm 14^{*}$ \\
\hline \multirow[t]{3}{*}{ Zym. Stim. Chemi. } & Control & $0.53 \pm .08$ & $0.50 \pm .05$ & $0.5 \mathrm{I} \pm .05$ \\
\hline & UFTiO2 high & $4.4 \pm .51^{*}$ & $4.4 \pm .47^{*}$ & $4.2 \pm .48^{*}$ \\
\hline & FTiO $_{2}$ high & $2.6 \pm .26^{*}$ & $2.8 \pm .35^{*}$ & $1.4 \pm .27$ \\
\hline \multirow[t]{3}{*}{ NO Dep. Chemi. } & Control & $4.3 \pm .06$ & $0.45 \pm .11$ & $0.39 \pm .08$ \\
\hline & UFTiO2 high & $3.3 \pm .1 I^{*}$ & $2.2 \pm .27^{*}$ & $1.0 \pm .12^{*}$ \\
\hline & FTiO $_{2}$ high & $2.6 \pm .20 *$ & $0.28 \pm .04$ & $0.230 \pm .04$ \\
\hline
\end{tabular}

Comparison of pulmonary toxicity parameters measured in control animals and for animals receiving the high doses (1.04 and $21.41 \mathrm{mg} / \mathrm{rat})$ of $\mathrm{UFTiO}_{2}$ and $\mathrm{FTiO}_{2}$ at all post-exposure time points. * denotes that the change from control is statistically significant $(\mathrm{p}<0.05)$ using ANOVA.

between the $\mathrm{UFTiO}_{2}$ and $\mathrm{FTiO}_{2}$ dose response curves at 1 day post-exposure.

\section{Pulmonary fibrosis}

At 42 days post-exposure, lungs were evaluated for pulmonary fibrosis by histological examination of lung tissue stained with Masson's trichrome. No excess fibrotic tissue was noted in lungs exposed to either $\mathrm{UFTiO}_{2}$ or $\mathrm{FTiO}_{2}$ (micrographs not shown).

\section{Comparison of lung burden data from $\mathrm{TiO}_{2}$ metal analysis} Rats were exposed to the medium dose of $\mathrm{UFTiO}_{2}$ or $\mathrm{FTiO}_{2}(0.52 \mathrm{mg} / \mathrm{rat}$ or $10.7 \mathrm{mg} / \mathrm{rat}$, respectively). This mass exposure resulted in the same particle surface area delivered to the lung $\left(0.06 \mathrm{~cm}^{2}\right.$ particles $/ \mathrm{cm}^{2}$ alveolar epithelial surface). The amount of $\mathrm{UFTiO}_{2}$ or $\mathrm{FTiO}_{2}$ in the lung was determined at 7 day and 42 day post-exposure. The metal analysis results indicate that the amount of $\mathrm{UFTiO}_{2}$ remaining in the lung decreased by $51 \%$ from the
7 day to the 42 day post-exposure time period, while the lung burden of $\mathrm{FTiO}_{2}$ decreased by $12 \%$ (Table 3 ).

\section{Comparison of lymph node burden data from $\mathrm{TiO}_{2}$ metal analysis}

To determine if the decline in lung burden was due to migration of particles to the lymph nodes, the amount of $\mathrm{TiO}_{2}$ present in the tracheo-bronchial (TBL) and thymic lymph nodes (TLN) was assessed at days 7 and 42 post-

Table 3: Decline in lung burden from 7 to 42 days post exposure

\begin{tabular}{lc}
\hline Particle & Percent decline \\
\hline $\mathrm{UFTiO}_{2}$ & $51 \pm 4 \%$ \\
\hline $\mathrm{FTiO}_{2}$ & $12 \pm 1 \%$
\end{tabular}

Values are for medium dose exposures $(0.52 \mathrm{mg} / \mathrm{rat} \mathrm{vs} 10.7 \mathrm{mg} / \mathrm{rat}$ for $\mathrm{UFTiO}_{2}$ and $\mathrm{FTiO}_{2}$, respectively, i.e., a surface area dose of $0.06 \mathrm{~cm}^{2}$ particles $/ \mathrm{cm}^{2}$ alveolar epithelial surface). Values are means $\pm \mathrm{SE}$ of at least 4 experiments. 
exposure. The results indicate that lymph node burden for $\mathrm{UFTiO}_{2}$ from 7 to 42 days post-exposure increased by $246 \%$, while a $134 \%$ increase was observed for $\mathrm{FTiO}_{2}$ (Table 4). Therefore, although relative migration of $\mathrm{UFTiO}_{2}$ to the lymph nodes exceeded that for $\mathrm{FTiO}_{2}$, this difference could not account for the much lower lung clearance of $\mathrm{FTiO}_{2}$ relative to $\mathrm{UFTiO}_{2}$ (Table 3).

\section{Comparison of lavaged lung burden data from $\mathrm{TiO}_{2}$ metal analysis}

Lung burden of $\mathrm{TiO}_{2}$ was measured in both lavaged and unlavaged lungs at 7 and 42 days post-exposure. The amount of $\mathrm{TiO}_{2}$ present in the lavaged lung is the non-lavagable fraction, i.e., the fraction not phagocytized by lavagable alveolar macrophages or present as free particles in the airspaces. The non-lavagable fraction would be expected to increase if particles migrated to the interstitium. The non-lavagable $\mathrm{UFTiO}_{2}$ or $\mathrm{FTiO}_{2}$ present in the lavaged lung increased from 7 to 42 days post-exposure (Table 5). However, there was a striking difference between the lavagable vs non-lavagable component of $\mathrm{UFTiO}_{2}$ compared to $\mathrm{FTiO}_{2}$. With $\mathrm{UFTiO}_{2}$, the majority of the particles were non-lavagable, suggesting migration to the interstitium. In contrast, the majority of $\mathrm{FTiO}_{2}$ was found in lavagable alveolar macrophages at both 7 and 42 days post-exposure.

\section{Discussion}

Fine $\mathrm{TiO}_{2}$ particles as well as other low-solubility, low-toxicity particles have long been of interest in particle toxicology. Historically, fine $\mathrm{TiO}_{2}$ particles were utilized as negative controls in numerous pulmonary toxicity studies. However, the current study shows that $\mathrm{FTiO}_{2}$ at relatively high mass lung burdens can induce pulmonary inflammation and damage, which are sustained through 42 days post-exposure. Using a mass dose metric, $\mathrm{UFTiO}_{2}$ was significantly more potent (at least 41 fold) than $\mathrm{FTiO}_{2}$ in causing sustained lung damage and inflammation. When exposure dose was normalized to equivalent surface area of particles delivered, no significant difference in potency between $\mathrm{UFTiO}_{2}$ and $\mathrm{FTiO}_{2}$ was observed. These findings help support the hypothesis that surface area of particles administered may be the more appropriate dose

Table 4: Increase in lymph node burden from 7 to $\mathbf{4 2}$ days postexposure

\begin{tabular}{lc}
\hline Particle & Percent increase \\
\hline $\mathrm{UFTiO}_{2}$ & $246 \pm 118 \%$ \\
\hline $\mathrm{FTiO}_{2}$ & $134 \pm 2 \%$
\end{tabular}

Values are for medium dose exposures $(0.52 \mathrm{mg} /$ rat vs $10.7 \mathrm{mg} / \mathrm{rat}$ for $\mathrm{UFTiO}_{2}$ and $\mathrm{FTiO}_{2}$, respectively, i.e., a surface area dose of $0.06 \mathrm{~cm}^{2}$ particles $/ \mathrm{cm}^{2}$ alveolar epithelial surface). Values are means \pm SE of at least 4 experiments.
Table 5: Percent lavagable vs non-lavagable TiO2 in the lung

\begin{tabular}{|c|c|c|}
\hline Particle & 7 Days post & 42 Days post \\
\hline \multirow[t]{2}{*}{$\mathrm{UFTiO}_{2}$} & 19\% lavagable & $0 \%$ lavagable \\
\hline & $81 \%$ non-lavagable & $100 \%$ non-lavagable \\
\hline \multirow[t]{2}{*}{$\mathrm{FTiO}_{2}$} & $91 \%$ lavagable & $84 \%$ lavagable \\
\hline & $9 \%$ non-lavagable & $16 \%$ non- lavagable \\
\hline
\end{tabular}

Values are for medium dose exposures $(0.52 \mathrm{mg} / \mathrm{rat}$ vs $10.7 \mathrm{mg} / \mathrm{rat}$ for $\mathrm{UFTiO}_{2}$ and $\mathrm{FTiO}_{2}$, respectively, i.e., a surface area dose of $0.06 \mathrm{~cm}^{2}$ particles $/ \mathrm{cm}^{2}$ alveolar epithelial surface).

metric for nanoparticle pulmonary toxicity studies. This conclusion, although not novel, has been recently disputed $[12,13]$.

Two similar studies were conducted by Warheit et al. $[12,13]$ to assess the lung toxicity of intratracheally instilled nano-sized and fine-sized $\mathrm{TiO}_{2}$. In both studies, they instilled fine and ultrafine $\mathrm{TiO}_{2}$ suspended in PBS into rats $(1$ or $5 \mathrm{mg} / \mathrm{kg}$ ) and compared measured pulmonary parameters to animals instilled with crystalline silica (used as a positive control). They monitored inflammation as PMN influx and cytotoxicity as LDH activity at 1 day, 1 week, 1 month and 3 months post-exposure in both studies. Warheit et al. $[12,13]$ concluded that exposures to nanosized $\mathrm{TiO}_{2}$ had no significant long term adverse pulmonary effects. They also reported similar acute levels of inflammation and cytotoxicity for both nano-sized and fine-sized $\mathrm{TiO}_{2}$, with only the highest dose $(5 \mathrm{mg} / \mathrm{kg})$ at 1 day post-exposure, causing a significant increase in inflammation for both nano- and finesized $\mathrm{TiO}_{2}$ exposures. Warheit and colleagues argued that the transient, reversible inflammation seen at 1 day postexposure with both nano-sized and fine-sized $\mathrm{TiO}_{2}$ was due primarily to the method of exposure (intratracheal instillation) rather than the effects of the nano-sized particles in the lung. The inflammation shown at 1 day postexposure in the Warheit et al. [12,13] studies quickly resolved to control levels by 1 week post-exposure.

In the first study, Warheit et al. [12] concluded that their results "run counter to the postulation that surface area is a major factor associated with the pulmonary toxicity of nanoscale particle-types". They argued that their study was instrumental in demonstrating that the fact that nanoparticles have larger surface areas does not necessarily indicate that they produce increased pulmonary inflammation and cytotoxicity when compared to fine-sized particles of similar composition. Warheit et al. [13], using very similar data to the Warheit et al. [12] study, expanded on the conclusions from the initial study. They again concluded that the fact that nanoparticles have larger surface areas does not necessarily indicate that they produce increased pulmonary inflammation and cytotoxicity 
when compared to fine-sized particles of similar composition.

The findings of the current study disagree with the findings of Warheit et al. [12,13]. The results of the current study indicate that surface area of the nanoparticles does in fact play a role in pulmonary toxicity, and that low mass burdens (as low as $0.26 \mathrm{mg} /$ rat) of $\mathrm{UFTiO}_{2}$ can cause persistent lung damage and inflammation over a 42 day post-exposure study period. The results of the current study support the fact that on a mass dose basis nanosized particles are more toxic than fine-sized particle of the same composition. However, when dose is normalized to surface area of particles administered, the difference in toxicity responses between $\mathrm{UFTiO}_{2}$ and $\mathrm{FTiO}_{2}$ for all pulmonary parameters measured becomes substantially less. This, therefore, indicates that surface area of particles is a critical driver of toxicity and may be an appropriate dose metric for pulmonary toxicity studies. The discrepancies between the two studies are believed to be due to the fact that Warheit et al. $[12,13]$ reports extreme agglomeration of the PBS-suspended nano-sized $\mathrm{TiO}_{2}$ utilized in their studies. In addition, significant agglomeration was also reported for the suspension of fine $\mathrm{TiO}_{2}$. This agglomeration issue leads to an over-estimation of delivered surface area of the particles. Indeed, their dynamic light scattering data indicate that once suspended in PBS both ultrafine and fine $\mathrm{TiO}_{2}$ had agglomerated particle sizes of $>2 \mu \mathrm{m}$. One would argue that since delivered particle size was equivalent, toxicity would not be expected to differ. In the present study, efforts were made to substantially improve the dispersion of $\mathrm{UFTiO}_{2}$ and $\mathrm{FTIO}_{2}$ by using rat bronchoalveolar lavage fluid (BALF) as the suspension medium. Results from our laboratory indicate that dispersion was vastly improved for both $\mathrm{UFTiO}_{2}$ and $\mathrm{FTiO}_{2}$ in BALF compared to PBS [14]. Our laboratory has reported that the diameter determined by dynamic light scattering of $\mathrm{UFTiO}_{2}$ suspended in a BALF model medium was $204 \mathrm{~nm}$ [16]. Therefore, in the present study, a potency difference between $\mathrm{UFTiO}_{2}$ and $\mathrm{FTiO}_{2}$ (on a mass basis) was observed because the physical surface areas of the UF vs F structures were different.

A recent study by Duffin et al. [17] further supports the conclusions of the current study. They investigated the relationship between inflammation, as indicated by neutrophil content of the bronchoalveolar lavage of rats 1824 hours after particle instillation, and particle size. Rats were instilled with ultrafine and fine-sized carbon black or titanium dioxide and then assessed for PMN influx into the lung. Duffin et al. [17] reported that the extent of inflammation was a function not of mass dose instilled but of the surface area of particles instilled. Likewise, the findings of the current study support the conclusion that inflammatory potential of $\mathrm{TiO}_{2}$ is a function of surface area of the particles administered.

Secondly, studies by Warheit et al. $[12,13]$ report that $\mathrm{UFTiO}_{2}$ at lung burdens of $1-5 \mathrm{mg} / \mathrm{kg}$ did not cause persistent lung damage and inflammation. In contrast, data from the present study indicate that lung burdens as low as $1 \mathrm{mg} / \mathrm{kg} \mathrm{UFTiO}_{2}$ caused significant lung damage and inflammation which persisted through 42 days post-exposure. In the present study, efforts were made to improve dispersion of $\mathrm{UFTiO}_{2}$ by using BALF as the suspension medium, as reported previously [14]. Shvedova et al. [18] reported that improved dispersion of ultrafine carbon black (UFCB) particles in BALF increased the inflammatory and damage potency compared to UFCB suspended in PBS; i.e., intratracheal instillation of a 30 fold greater mass of poorly dispersed UFCB suspended in PBS was required to attain the same level of pulmonary damage (LDH activity) and inflammation (PMN level) as well dispersed UFCB suspended in BALF in a rat model. Therefore, poor dispersion of $\mathrm{UFTiO}_{2}$ in PBS (diameters of over $2 \mu \mathrm{m}$ determined by dynamic light scattering) may account for the low biological activity previously reported by Warheit et al. [12,13].

Warheit et al. [13] suggested that particle characteristics, such as surface reactivity, crystal structure, surface $\mathrm{pH}$, surface coating, shape, etc., may play important roles in the pulmonary toxicity of nanoparticles. They have demonstrated that $\mathrm{UFTiO}_{2}$ in the uncoated, $80 / 20$ anatase/rutile form exhibited greater surface activity than aluminacoated rutile as judged by ability to deplete vitamin $\mathrm{C}$ in an acellular assay. In addition, Sayes et al. [19] demonstrated that 80/20 anatase/rutile generated 6 fold more reactive species (measured as acellular luminol-enhanced chemiluminescence) than rutile after UV irradiation. They argued that this increased surface reactivity resulted in greater in vitro cytotoxicity for the anatase form of $\mathrm{UFTiO}_{2}$. In the present study, the $\mathrm{UFTiO}_{2}$ used was $80 / 20$ anatase/ rutile, while the $\mathrm{FTiO}_{2}$ was the rutile crystal form. However, the particle samples were not irradiated with UV light in this study. In the absence of UV irradiation, Sayes et al. [19] reported the $\mathrm{UFTiO}_{2}$ in the $80 / 20$ anatase/rutile form generated only twice the reactive species as ultrafine rutile. Such as small difference in surface reactivity can not account for the 40 fold greater potency of ultrafine vs. fine $\mathrm{TiO}_{2}$ on an equal mass basis reported in the present study. However, a 2 fold difference in surface activity may explain why normalizing dose to equivalent particle surface area delivered did not result in identical potency for the ultrafine and fine particle samples. Recent results indicate that the shape of nanoparticles can affect toxic potential. Porter et al. [20] demonstrated that $\mathrm{TiO}_{2}$ nanowires exhibited greater toxicity in vitro and greater inflammatory potential in vivo than $\mathrm{TiO}_{2}$ nanospheres of the same crystal 
structure and diameter. Therefore, although the present study concentrated on the role of particle surface area in nanoparticle toxicity, results do not discount the importance of other particle characteristics in nanoparticle toxicity.

Results of lung burden studies suggest that although a large portion of $\mathrm{FTiO}_{2}$ appears to have been engulfed by lavagable alveolar macrophages (Table 5) clearance of $\mathrm{FTiO}_{2}$ was relatively low, $12 \%$ from 7 to 42 days postexposure (Table 3 ). One, thus, could conclude that the lungs were in overload at this $\mathrm{FTiO}_{2}$ burden $(10.7 \mathrm{mg} / \mathrm{rat})$. As proposed by Morrow [10], when alveolar macrophages have engulfed a large volume of particles, the phagocytes become immobile and clearance fails. In contrast, macrophage clearance apparently is relatively robust after exposure to $0.52 \mathrm{mg} /$ rat $\mathrm{UFTiO}_{2}$; i.e., $51 \%$ decline in lung burden is observed from 7 to 42 days post-exposure (Table 3). This clearance is only partially due to migration of $\mathrm{UFTiO}_{2}$ to the lymph nodes (Table 4). In addition, migration of $\mathrm{UFTiO}_{2}$ into the alveolar walls is far more substantial than that for $\mathrm{FTiO}_{2}$ (Table 5). A high rate of interstitilization of $\mathrm{UFTiO}_{2}$ vs $\mathrm{FTiO}_{2}$ has been reported previously [21].

\section{Conclusion}

In summary, exposure of rats by intracheal instillation to a well-dispersed suspension of $\mathrm{UFTiO}_{2}$ caused dose dependent pulmonary damage and inflammation, which persisted 42 days post-exposure. On a mass dose basis, $\mathrm{UFTiO}_{2}$ was at least 41 fold more potent than $\mathrm{FTiO}_{2}$. When exposure dose was normalized to surface area of particles delivered, this potency difference was no longer significant. Burden data indicate that $\mathrm{UFTiO}_{2}$ migrates to the interstitium to a far greater extent than $\mathrm{FTiO}_{2}$. Results support the use of particle surface area as a dose metric for evaluation of pulmonary response to particles.

\section{Methods}

\section{Experimental design}

Recently, the hypothesis that nanoparticles exhibit unique bioactivity due to their large surface area per mass has been questioned $[12,13]$. The aim of the present study was to readdress the issue of the relative toxicity of $\mathrm{UFTiO}_{2}$ vs $\mathrm{FTiO}_{2}$ on a mass dose or equivalent particle surface area delivered dose basis. In this study, particle dispersion was substantially improved by suspension of particles in diluted alveolar lining fluid obtained by bronchoalveolar lavage of naïve rats as described previously [14]. Rats were exposed to various mass concentrations of $\mathrm{UFTiO}_{2}$ vs $\mathrm{FTiO}_{2}$ by intratracheal instillation. Mass doses of $\mathrm{UFTiO}_{2}$ (0.26, 0.52 and $1.04 \mathrm{mg} / \mathrm{rat})$ and $\mathrm{FTiO}_{2}$ (5.35, 10.7 and $21.41 \mathrm{mg} / \mathrm{rat})$ were chosen to result in equivalent total particle surface area delivered doses $(0.0313,0.0625$ and $0.125 \mathrm{~cm}^{2}$ of particles administered per $\mathrm{cm}^{2}$ of alveolar epithelial surface) for $\mathrm{UFTiO}_{2}$ and $\mathrm{FTiO}_{2}$. Rats were sacri- ficed at 1 day, 7 days, and 42 days post-exposure, and markers of inflammation (PMN number or inflammatory mediator levels in BAL samples), lung injury (LDH activity or albumin levels in BAL samples), and macrophage activity (zymosan-stimulated or NO-dependent chemiluminescence) were monitored. The magnitude of pulmonary responses to exposure to $\mathrm{UFTiO}_{2}$ vs $\mathrm{FTiO}_{2}$ was then compared on a mass dose and an equivalent surface area of particles administered dose basis to evaluate the role of particle surface area in the pulmonary response.

\section{Animals for in vivo exposures}

The rats used for the in vivo experiments were male Fischer CDF (F344/DuCrl) rats weighing 200-300 g ( 10 weeks old at arrival) obtained from Charles River (Raleigh, NC). The animals were housed in an AAALAC-accredited; specific pathogen-free, environmentally controlled facility. The animals were monitored to be free of endogenous viral pathogens, parasites, mycoplasms, Helicobacter and CAR Bacillus. Animals were housed in ventilated cages which were provided HEPA-filtered air, with Alpha-Dri virgin cellulose chips and hardwood Beta-chips used as bedding. The rats were maintained on a Teklad 2918 diet and tap water, both of which were provided ad libitum.

\section{Bronchoalveolar fluid collection for particle suspension media}

Rats were euthanized with an i.p. injection of sodium pentobarbital (>100 mg/kg body weight) and exsanguinated by cutting the descending aorta. A tracheal cannula was inserted and bronchoalveolar lavage was conducted [22]. A $6 \mathrm{ml}$ aliquot of cold $\mathrm{Ca}^{+2}$ and $\mathrm{Mg}^{+2}$-free phosphatebuffered saline (PBS) was used for the lavage wash. The cold PBS was flushed into and out of the lungs two times before the lavage fluid was collected. The bronchoalveolar lavage (BAL) from five rats was combined and centrifuged at $600 \times \mathrm{g}$ for 10 minutes using a Sorvall RC 3B Plus centrifuge (Sorvall Thermo Electron Corporation, Asheville NC). The supernatant was decanted into a new tube, while the pellet was discarded. This acellular bronchoalveolar lavage fluid (BALF) was then used as the vehicle for particle suspensions. The BALF was collected fresh the same day that the particulate suspensions were made.

\section{Particles}

UFTiO $_{2}$ (Aeroxide $\mathrm{TiO}_{2}$ P-25) was obtained as a gift from Degussa Corporation (Parsippany, NJ). UFTiO 2 had a primary particle size of $21 \mathrm{~nm}$ and was a 80/20 mixture of anatase/rutile. $\mathrm{FTiO}_{2}$ (titanium (IV) oxide, \#224227) was purchased from Sigma-Aldrich (St. Louis, MO). $\mathrm{FTiO}_{2}$ had a primary particle size of $1 \mu \mathrm{m}$ and was $100 \%$ rutile.

\section{Suspension of $\mathrm{UFTiO}_{2}$ and $\mathrm{FTiO}_{2}$ in rat $\mathrm{BALF}$}

Ultrafine titanium dioxide $\left(\mathrm{UFTiO}_{2}\right)$ and fine titanium dioxide $\left(\mathrm{FTiO}_{2}\right)$ particles were sieved using a Retsch AS 200 Sieve (Retsch GmbH, Haann, Germany) through 1.18 
mm, $250 \mu \mathrm{m}$, and $45 \mu \mathrm{m}$ mesh screens. Particles were weighed and suspended in rat BALF to obtain the desired concentration. Once the particles were added to the BALF, the suspensions were pulse sonicated with 5 individual pulses at a duty cycle setting of $10 \%$ and an output setting of 1 with a Branson 450 Sonifier probe sonicator (Branson Ultrasonics Corporation, Danbury CT.). This method has been reported previously by our laboratory to result in well dispersed suspensions as determined by light and electron microscopy [14]. Dynamic light scattering of $\mathrm{UFTiO}_{2}$ suspended in a BALF model medium indicated that ultrafine particles had a diameter of $204 \pm 18 \mathrm{~nm}$ [16].

\section{In vivo exposures}

To receive their respective dose of particles, each rat was anesthetized with an intraperitoneal (i.p.) injection of methohexital sodium (30-40 mg/kg body weight; Monarch Pharmaceuticals, Bristol, TN). Each animal was then instilled via intratracheal instillation (IT) using a 20-gauge 4 -inch ball tipped animal feeding needle [22]. Each animal was instilled with $0.3 \mathrm{ml}$ of $\mathrm{UFTiO}_{2}$ or $\mathrm{FTiO}_{2}$ suspended in BALF. BALF $(0.3 \mathrm{ml})$ was instilled as the control. Results from our laboratory indicate that instillation of BALF does not cause lung inflammation [14]. In addition, suspension of quartz in BALF did not alter its inflammatory potency [14].

\section{Bronchoalveolar lavage and cell differentials}

At 24-hours, 7 days, and 42 days post-IT, the animals were euthanized with an i.p. injection of sodium pentobarbital (>100 mg/kg body weight) and exsanguinated by cutting the descending aorta. A tracheal cannula was inserted and bronchoalveolar lavage (BAL) was conducted [22]. A $6 \mathrm{ml}$ aliquot of cold $\mathrm{Ca}^{+2}$ and $\mathrm{Mg}^{+2}$ free PBS was used for the first lavage wash. The cold PBS was flushed into and out of the lungs two times before the lavage fluid was collected. After the first lavage wash was collected and stored separately, the BAL continued with $8 \mathrm{ml}$ aliquots of cold $\mathrm{Ca}^{+2}$ and $\mathrm{Mg}^{+2}$-free PBS until an additional $80 \mathrm{ml}$ of bronchoalveolar lavage was collected. The BAL was then centrifuged at $600 \times \mathrm{g}$ for 10 minutes using a Sorvall RC 3B Plus centrifuge (Sorvall Thermo Electron Corporation, Asheville NC). After centrifugation, the supernatant from the first lavage wash was decanted into a clean conical vial and was stored on ice to be used for lung damage/cytotoxicity analysis. The remaining supernate from the lavages was discarded, and the cells remaining were washed with cold $\mathrm{Ca}^{+2}$ and $\mathrm{Mg}^{+2}$-free PBS and spun again at $600 \times \mathrm{g}$ for 10 minutes. After this, the supernatant was discarded and the cells were resuspended in $1 \mathrm{ml}$ of HEPES-buffered medium. Using these lavage samples, polymorphonuclear neutrophil (PMN) and alveolar macrophage (AM) counts were determined to assess inflammation. The number of $\mathrm{AM}$ and PMN was determined according to their unique cell diameters, using an electronic cell counter equipped with a cell sizing attachment (Beckman Coulter Multisizer 3 Counter, Hialeah, FL).

\section{BAL fluid lactate dehydrogenase activity and albumin concentration}

The degree of cytotoxicity induced by the instilled particles was determined by lactate dehydrogenase (LDH) activity in the BALF. LDH activity was measured using a Roche COBAS MIRA Plus chemical analyzer (Roche Diagnostic Systems Inc., Branchburg, NJ) as described previously by our laboratory [22]. Albumin concentrations in BALF were assessed to examine if instilled particle exposures had compromised the integrity of the alveolar air/ blood barrier. Albumin concentrations were also measured using a Cobas Fara II Analyzer (Roche Diagonostic Systems, Montclair, NJ) as previously described by our laboratory [22].

\section{Mediator measurements in bronchoalveolar lavage fluid}

The presence of inflammatory mediators in the BALF was analyzed by enzyme-linked immunosorbent assay (ELISA). The levels of mediators present were measured using commercially available ELISA kits (BioSource International Inc., Camarillo, CA). Three mediators were quantified: tumor necrosis factor- $\alpha$ (TNF- $\alpha$ ), interleukin (IL)- $\beta$, and macrophage-inflammatory protein-2 (MIP-2).

\section{Zymosan-stimulated and NO-dependent alveolar macrophage chemiluminescence}

Reactive oxygen species production was determined by measuring AM chemiluminescence. According to Van Dyke et al. [23], only AM will generate reactive oxygen species in response to unopsonized zymosan in the chemiluminecence assay procedure. The AM chemiluminescence assay was conducted in the same manner as previously described by our laboratory [22]. Briefly, resting AM chemiluminescence was determined by incubating $1.0 \times 10^{6} \mathrm{AM} / \mathrm{ml}$ at $37^{\circ} \mathrm{C}$ for 20 minutes, followed by the addition of 5-amino-2,3-dihydro-1,4, phthalazinedione (luminol) to a final concentration of $0.08 \mu \mathrm{g} / \mathrm{ml}$. This was then followed by the measurement of chemiluminescence generated over 15 minutes.

Zymosan-stimulated chemiluminescence was determined by adding unopsonized zymosan $(2 \mathrm{mg} / \mathrm{ml})$ to the AM samples immediately prior to measurement of chemiluminescence. NO-dependent chemiluminescence was determined by adding the unopsonized zymosan as well as N-nitro-L-arginine methyl ester HCL (L-NAME) to the AM samples immediately prior to measurement of chemiluminescence. Zymosan-stimulated (zymosan - rest) and NO-dependent (zymosan - zymosan with L-NAME) chemiluminescence were both measured using a Berthold automated luminometer (Berthold Autolumat LB 953, 
EG\&G, Gaithersburg, MD) at 390-620 nm for $15 \mathrm{~min}-$ utes.

\section{Tissue collection and analysis for titanium dioxide burden analysis}

Immediately after the 7 and 42- day exposure time periods, rats were euthanized with an i.p. injection of sodium pentobarbital (>100 mg/kg body weight). The left and right lungs as well as the tracheobronchial (TBL) and thymic (TLN) lymph nodes were removed for titanium dioxide burden analysis. After removal, the lungs and lymph nodes were weighed and then frozen at $-80^{\circ} \mathrm{C}$. The lungs and lymph nodes were then lyophilized. The lyophilized samples were sent to Bureau Veritas North America, Inc. (Novi, MI) for $\mathrm{TiO}_{2}$ metal analysis.

\section{Histopathology}

Immediately after the 7 and 42 days exposure, rats were euthanized with i.p. injection of sodium pentobarbital (> $100 \mathrm{mg} / \mathrm{kg}$ body weight). The lungs were removed and infused to $25 \mathrm{~cm} \mathrm{H}_{2} \mathrm{O}$ with $10 \%$ buffered neutral formalin. Paraffin-embedded histologic sections were stained with hematoxylin and eosin (H\&E) or Masson's trichrome for light microscopic examination by a board-certified veterinary pathologist.

\section{Statistics}

Statistical differences between control groups and treatment groups for the in vivo experiments examining the toxicity of titanium dioxide were determined using an analysis of variance (ANOVA). Individual means were compared using the Student-Newman-Keuls Method multiple comparison procedure with an overall significance level of $\mathrm{p}=0.05$. A linear regression curve analysis with a 95\% confidence interval was also conducted on the surface area data for each pulmonary parameter measured.

\section{Disclaimer}

The findings and conclusions in this report are those of the authors and do not necessarily represent the views of the National Institute for Occupational Safety and Health.

\footnotetext{
Abbreviations

AAALAC: Association for Assessment and Accreditation of Laboratory Animal Care; AM: Alveolar macrophage; ANOVA: Analysis of variance; BAL: Bronchoalveolar lavage; BALF: Bronchoalveolar lavage fluid; BET: BrunauerEmmett-Teller; $\mathrm{Ca}^{+2}$ : Calcium; $\mathrm{CB}$ : Carbon black; cm: centimeter; CVF: Certified virus free; FCB: Fine carbon black; FTiO2: Fine titanium dioxide; H\&E: Hematoxylin and eosin; IL: Interleukin; i.p.: intraperitoneal; IT: Intratreacheal instillation; LDH: Lactate dehydrogenase; $\mathrm{Mg}^{+2}$ : Magnesium; $\mu \mathrm{m}$ : Micrometer; mg: Milligram; MIP: Macrophage inflammatory protein; ml: Milliliter; SE: Standard
}

error; TBL: Tracheo-bronchial lymph node; $\mathrm{TiO}_{2}$ : Titanium dioxide; TLN: Thymic lymph node; TNF: Tumor necrosis factor; UF: Ultrafine; UFCB: Ultrafine carbon black; $\mathrm{UFTiO}_{2}$ : Ultrafine titanium dioxide.

\section{Competing interests}

The authors declare that they have no competing interests.

\section{Authors' contributions}

TMS carried out all of the in vivo experiments involved in this study including the intratracheal instillations and animal sacrifices. TMS drafted the manuscript and performed the statistical analysis. CK conducted the histopathology analysis for the study. Both TMS and VC conceived of the study and participated in its design. VC participated in the study coordination, data analysis and interpretation, and helped draft the manuscript. All authors read and approved the final manuscript.

\section{References}

I. McAllister K, Sazani P, Adam M, Cho M, Rubinstein M, Samuluski R: Polymeric nanogels produced via inverse microemulsion polymerization as potential gene and antisense delivery agents. J Am Chem Soc 2002, 124: I51 98-15207.

2. Grassian V, O'Shaughnessy P, Adamcakova-Dodd A, Pettibone J: Inhalation exposure study of titanium dioxide nanoparticles with a primary particle size of $\mathbf{2}$ to $\mathbf{5} \mathbf{~ n m}$. Environ Health Perspect 2007, I I 5(3):397-402.

3. Donaldson K, Li XY, MacNee W: Ultrafine (nanometer) particle mediated lung injury. J Aerosol Sci 1998, 29:553-60.

4. Oberdorster G: Significance in parameters in the evaluation of exposure-dose response relationships of inhaled particles. Inhal Toxicol 2002:73-89.

5. Maynard A, Zimmer T: Evaulation of grinding aerosols in terms of alveolar dose: the significance of using mass, surface area and number metrics. Ann Occup Hyg 2002, 46(SuppI I):3 I 5-319.

6. ILSI Risk Science Institute Workshop Participants: The relevance of the rat lung response to particle overload for human risk assessment: A workshop consensus report. Inhal Toxicol 2000, I2(I-2): I- 17

7. Ferin J, Oberdorster G, Penney D: Pulmonary retention of ultrafine and fine particles in rats. Am J Respir Cell Mol Biol 1992, 6(5):535-542.

8. Donaldson K, Brown D, Clouter A, Duffin R, MacNee W, Renwick L, Tran $L$, Stone $V$ : The pulmonary toxicology of ultrafine particles. J Aerosol Med 2002, I 5(2):2 I 3-220.

9. Monteiller C, Tran L, MacNee W, Faux S, Jones A, Miller B, Donaldson $\mathrm{K}$ : The pro-inflammatory effects of low-toxicity low-solubility particles, nanoparticles and fine particles, on epithelial cells in vitro: the role of surface area. Occup Environ Med 2007, 64:609-615.

10. Morrow P: Possible mechanism to explain dust overloading of the lungs. Fundam Appl Toxicol 1988, 10:369-384.

II. Tran L, Buchanan R, Cullen R: Inhalation of poorly soluable particles II. Influence of particle surface area on inflammation and clearance. Inhal Toxicol 2000, I 2: I | | $3-1 \mid 26$.

12. Warheit $D$, Webb T, Sayes $C$, Colvin V, Reed K: Pulmonary instillation studies with nanoscale $\mathrm{TiO}_{2}$ rods and dots in rats: toxicity is not dependent upon particle size and surface area. Toxicol Sci 2006, 91 ( I):227-236.

13. Warheit D, Webb T, Reed K, Frerichs S, Sayes C: Pulmonary toxicity study in rats with three forms of ultrafine- $\mathrm{TiO}_{2}$ particles: differential responses related to surface properties. Toxicology 2007, 230(I):90-104.

14. Sager T, Porter D, Robinson V, Lindsley W, Schwegler-Berry D, Castranova $\mathrm{V}$ : An improved method to disperse nanoparticles for in vitro and in vivo investigation of toxicity. Nanotoxicol 2007, I:118-129. 
15. Brunauer S, Emmett $P$, Teller E: Adsorption of gases in multimolecular layers. J Am Chem Soc 1938, 60:309-319.

16. Porter D, Sriram K, Wolfarth M, Jefferson A, Schwegler-Berry D, Andrew M, Castranova V: A biocompatible medium for nanoparticle dispersion. Nanotoxicol 2008, 2: I44- 154.

17. Duffin R, Tran L, Brown D, Stone V, Donaldson K: Proinflammogenic effects of low-toxicity and metal nanoparticles in vivo and in vitro: highlighting the role of particle surface area and surface reactivity. Inhal Toxicol 2007, 19:849-856.

18. Shvedova AA, Sager T, Murray A, Kisin E, Porter DW, Leonard SS, Schwegler-Berry D, Robinson VA, Castranova V: Critical issues in the evaluation of possible effects resulting from airborne nanoparticles. In Nanotechnology: Characterization, Dosing and Health Effects Volume 14. Edited by: Monteiro-Riviere N, Tran L. Informa Healthcare, Philadelphia; 2007:22I-232.

19. Sayer CM, Wahi R, Kurian PA, Liu Y, Weat JL, Ausman KD, Warheit $D B$, Colvin VL: Correlating nanoscale titania structure with toxicity: a cytotoxicity and inflammatory response study with human dermal fibroblasts and human lung epithelial cells. Toxicol Sci 2006, 92: 174-185.

20. Porter DW, Holian A, Sriram K, Wu N, Wolfarth M, Hamilton R, Buford $M$ : Engineered titanium dioxide nanowire toxicity in vitro and in vivo. The Toxicologist 2008, 102:AI492.

21. Oberdorster G, Ferin J, Jehnert BE: Correlation between particle size, in vivo particle persistence, and lung injury. Environ Health Perspect 1994, 102(Suppl 5): 173-179.

22. Porter D, Hubbs A, Robinson V, Battelli L, Greskevitch M, Barger M, Landsittel D, Jones W, Castranova V: Comparative pulmonary toxicity of blasting sand and five substitute abrasive blasting agents. J Toxicol Environ Health A 2002, 65( I6): I I I I-I I 40.

23. Van Dyke K, Van Scott M, Castranova V: Measurement of phagocytosis and cell-mediated cytotoxicity by chemiluminescence. Methods Enzymol 1987:498-507.

Publish with Bio Med Central and every scientist can read your work free of charge

"BioMed Central will be the most significant development for disseminating the results of biomedical research in our lifetime. "

Sir Paul Nurse, Cancer Research UK

Your research papers will be:

- available free of charge to the entire biomedical community

- peer reviewed and published immediately upon acceptance

- cited in PubMed and archived on PubMed Central

- yours - you keep the copyright
BioMedcentral 